\title{
Impact of Blood Type, Functional Polymorphism (T-1676C) of the COX-1 Gene Promoter and Clinical Factors on the Development of Peptic Ulcer during Cardiovascular Prophylaxis with Low-Dose Aspirin
}

\author{
Pin-Yao Wang, ${ }^{1}$ Hsiu-Ping Chen, ${ }^{1}$ Angela Chen, ${ }^{1}$ Feng-Woei Tsay, ${ }^{2,3}$ \\ Kwok-Hung Lai, ${ }^{2,3}$ Sung-Shuo Kao, ${ }^{2,3}$ Wen-Chi Chen, ${ }^{2,3,4}$ Chao-Hung Kuo, ${ }^{5}$ \\ Nan-Jing Peng, ${ }^{3,6}$ Hui-Hwa Tseng, ${ }^{7}$ and Ping-I Hsu ${ }^{2,3}$ \\ ${ }^{1}$ Institute of Biomedical Sciences, National Sun Yat-Sen University, Kaohsiung 804, Taiwan \\ ${ }^{2}$ Division of Gastroenterology, Department of Internal Medicine, Kaohsiung Veterans General Hospital, 386 Ta Chung 1st Road, \\ Kaohsiung 813, Taiwan \\ ${ }^{3}$ School of Medicine, National Yang-Ming University, Taipei 112, Taiwan \\ ${ }^{4}$ Department of Chemistry, College of Science, National Kaohsiung Normal University, Kaohsiung 802, Taiwan \\ ${ }^{5}$ Division of Gastroenterology, Department of Internal Medicine, Kaohsiung Medical University Hospital, Kaohsiung 807, Taiwan \\ ${ }^{6}$ Department of Nuclear Medicine, Kaohsiung Veterans General Hospital and National Yang-Ming University, Kaohsiung 813, Taiwan \\ ${ }^{7}$ Department of Pathology, Kaohsiung Veterans General Hospital, Kaohsiung 813, Taiwan
}

Correspondence should be addressed to Angela Chen; achen@nsysu.edu.tw and Ping-I Hsu; williamhsup@yahoo.com.tw

Received 24 May 2014; Accepted 9 July 2014; Published 27 August 2014

Academic Editor: Seng-Kee Chuah

Copyright (C) 2014 Pin-Yao Wang et al. This is an open access article distributed under the Creative Commons Attribution License, which permits unrestricted use, distribution, and reproduction in any medium, provided the original work is properly cited.

Aims. To investigate the impact of blood type, functional polymorphism (T-1676C) of the COX-1 gene promoter, and clinical factors on the development of peptic ulcer during cardiovascular prophylaxis with low-dose aspirin. Methods. In a case-control study including 111 low-dose aspirin users with peptic ulcers and 109 controls (asymptomatic aspirin users), the polymorphism (T-1676C) of the COX-1 gene promoter was genotyped, and blood type, $H$ pylori status, and clinical factors were assessed. Results. Univariate analysis showed no significant differences in genotype frequencies of the COX-1 gene at position -1676 between the peptic ulcer group and control group. Multivariate analysis revealed that blood type $\mathrm{O}$, advanced age, history of peptic ulcer, and concomitant use of NSAID were the independent risk factors for the development of peptic ulcer with the odds ratios of the 2.1, 3.1, 27.6, and 2.9, respectively. Conclusion. The C-1676T polymorphism in the COX-1 gene promoter is not a risk factor for ulcer formation during treatment with low-dose aspirin. Blood type $\mathrm{O}$, advanced age, history of peptic ulcer, and concomitant use of NSAID are of independent significance in predicting peptic ulcer development during treatment with low-dose aspirin.

\section{Introduction}

Low-dose aspirin $(75-325 \mathrm{mg} /$ day $)$ is widely used in the prevention of myocardial infarction or ischemic stroke [1]. Besides the patients requiring secondary prevention of cardiovascular events, the American Heart Association recommends prophylactic aspirin for the subjects who have a 10year cardiovascular risk equal to or more than $10 \%$ [2].
Currently, approximately $36 \%$ of the adult US populationmore than 50 million people-is estimated to take aspirin regularly for cardiovascular disease prevention [3]. However, due to its inhibition of prostaglandin synthesis, direct cytotoxicity, and microvascular injury, aspirin is associated with upper gastrointestinal side effects, which range from mild dyspepsia to life-threatening bleeding and perforation from peptic ulcers [4]. Even at doses as low as $10 \mathrm{mg}$, aspirin 
has been shown to cause gastrointestinal damage and is associated with a greater risk of gastroduodenal ulcers and life-threatening ulcer complications [5].

Previous studies revealed that a history of ulcer, advanced age ( $>70$ years), concomitant use of nonsteroidal antiinflammatory drugs (NSAIDs), use of dual antiplatelet therapy, Helicobacter pylori (H. pylori) infection, and history of alcohol abuse were risk factors for gastroduodenal ulcer in low-dose aspirin users $[6,7]$. However, the genetic risk factors for the development of peptic ulcer in aspirin users remain unclear. A recent 35-year cohort study from Sweden also showed that individuals with blood group $\mathrm{O}$ had a higher risk of peptic ulcer development than those with other blood groups and blood group A was associated with the risk of developing gastric cancer [8]. Nonetheless, whether blood group $\mathrm{O}$ is also a risk factor for ulcer development in lowdose aspirin users remains to be clarified.

Cyclooxygenase-1 (COX-1) is a constitutively expressed enzyme that generates prostaglandins for gastrointestinal integrity. Aspirin can inhibit COX-1 enzyme and decrease prostaglandin synthesis of gastrointestinal tract. Recently, functional polymorphism (T-1676C) in the COX-1 gene promoter has been identified and was reported to alter putative transcription factor (GATA-1, CdxA) binding sites [9, 10]. Arisawa et al. revealed that the number of $-1676 \mathrm{~T}$ alleles of the COX-1 gene promoter was a significant risk factor for the NSAID-induced ulcer in Japan [11]. However, whether the COX-1 genetic polymorphism also plays an important role in the development of peptic ulcer in low-dose aspirin users remains unclear.

The aim of this case-control study was to investigate the clinical risk factors and genetic markers for the development of peptic ulcer during cardiovascular prophylaxis with lowdose aspirin.

\section{Patients and Methods}

2.1. Patients. A total of 111 consecutive low-dose (75$325 \mathrm{mg}$ /day) aspirin users with peptic ulcer, who attended the Kaohsiung Veterans General Hospital or Kaohsiung Medical University Hospital, were included in this study. The reason for choosing this dosage range of aspirin is because the common dose of aspirin used for the prevention of cardiovascular diseases varies between 75 and $325 \mathrm{mg}$ daily [1-3]. The diagnosis of peptic ulcer was confirmed by endoscopic examination. One hundred and nine consecutive asymptomatic low-dose aspirin users who underwent upper gastrointestinal endoscopy for endoscopic surveillance and had normal endoscopic appearance or gastritis only served as controls. The exclusion criteria included (1) consumption of proton pump inhibitor (PPI) or histamine-2 receptor antagonist within 2 weeks before endoscopy, (2) coexistence of gastrointestinal malignancies, (3) serious medical illness (e.g., decompensated liver cirrhosis, uremia, septic shock, acute stroke, and acute myocardial infection within 2 weeks), and (4) receiving low-dose aspirin less than 2 weeks. The study was approved by the Medical Research Committee of the Kaohsiung Veterans General Hospital and Kaohsiung
Medical University Hospital. All patients and controls gave informed consent.

2.2. Methods. Endoscopies were performed with the Olympus GIF XV10 and GIF XQ200 (Olympus Corp., Tokyo, Japan). During endoscopy, biopsies over antrum and body were performed for rapid urease test (with one specimen from the antrum and another one from the body). The rapid urease test was performed according to our previous studies [12]. Rapid urease tests were assessed by a technician, blind as to the endoscopic features. The diagnosis of $H$. pylori infection was based on the result of rapid urease test. An ulcer was defined as a circumscribed mucosal break $3 \mathrm{~mm}$ or more in diameter, with a well-defined ulcer crater in the stomach or duodenum [13]. The size of ulceration was measured by opening a pair of biopsy forceps of known span in front of the ulcer.

Before endoscopy, venous blood was drawn for $C O X-1$ genotyping. To assess the significance of clinical characteristics, the following data were recorded for each patient: age, sex, blood type, smoking, alcohol consumption, coffee consumption, tea consumption, previous history of peptic ulcer, and use of thienopyridine, coumadin, steroid, or NSAIDs within 2 weeks prior to endoscopy. Coffee or tea consumption was defined as drinking 1 cup or more per day.

2.2.1. COX-1 Genotyping. Genomic DNA was extracted from $3 \mathrm{~mL}$ of whole blood by the use of a QIAamp DNA Extraction Mini Kit (QIAGEN Inc., Valencia, CA). The COX-1 genotype (T-1676C) was determined using the restriction fragment length polymorphism (RFLP). The primer set (COX-1F, $5^{\prime}$-TGGACCAGTCCTCAGAGACC-3' ${ }^{\prime}$ and COX$1 \mathrm{R}, 5^{\prime}$-CCCATC AAGTCACCACACCT- $3^{\prime}$ ) [14] was used to amplify DNA fragments, of which the fragments of 243 base pairs were obtained. The PCR reactions were performed using 2.5 U of Taq DNA polymerase together with the corresponding Taq buffer supplemented with $2 \mathrm{mM} \mathrm{MgCl}_{2}$ (Invitrogen, Life Technologies, California, USA), $2.5 \mathrm{mM}$ each of the 4 deoxynucleotide triphosphates (dNTP, $100 \mathrm{mM}$, Amersham, $\mathrm{UK}), 0.5 \mathrm{M}$ of each amplification primer, and 40-100 ng of genomic DNA mixed up to a final volume of $25 \mu \mathrm{L}$. The PCR analyses were run on a PCR thermal cycler (model 2400, Perkin Elmer, Foster City, CA) with the following profile: 5 min of denaturation at $94^{\circ} \mathrm{C}$, followed by 35 cycles of $30 \mathrm{~s}$ at $94^{\circ} \mathrm{C}, 30 \mathrm{~s}$ at $64^{\circ} \mathrm{C}$, and $60 \mathrm{~s}$ at $72^{\circ} \mathrm{C}$, with a final elongation at $72^{\circ} \mathrm{C}$ for $7 \mathrm{~min}$. The amplified DNA fragments (243 base pairs) contained two $T s p R$ I restriction sites. After $T s p R$ I digestion, fragments with172 base pairs and 71 base pairs were observed for genotype TT; fragments with 172 base pairs, 88 base pairs, 84 base pairs, and 71 base pairs were observed for genotype TC; and fragments with 88 base pairs, 84 base pairs, and 71 base pairs were observed for genotype CC. Then the PCR products were digested by $T s p R$ I restriction enzymes in a reaction mixture $(20 \mu \mathrm{L})$ containing PCR products $(15 \mu \mathrm{L})$, $1 \mathrm{X}$ Buffer 4, 1X BSA, and TspR I $(0.5 \mathrm{U} / \mu \mathrm{L})$ at $65^{\circ} \mathrm{C}$ for 16 hours. The digested fragments were separated and analyzed by electrophoresis using 3\% agarose gel. Genotypes of COX-1 
TABLE 1: Clinical characteristics of aspirin users with and without peptic ulcer.

\begin{tabular}{lccc}
\hline & Gastritis/normal $(N=109)$ & Peptic ulcer $(N=111)$ & $P$ value \\
\hline Age & $67.0 \pm 12.1$ & $72.0 \pm 10.3$ & \\
Sex & & $31(27.9 \%)$ & $0.001^{*}$ \\
$\quad$ Female & $40(36.7 \%)$ & $80(72.1 \%)$ & 0.501 \\
$\quad$ Male & $69(63.3 \%)$ & $19(17.1 \%)$ & \\
Cigarette smoking & $13(11.9 \%)$ & $9(8.1 \%)$ & 0.275 \\
Heavy drinker & $7(6.4 \%)$ & $11(9.9 \%)$ & 0.630 \\
Coffee consumption & $22(20.2 \%)$ & $27(24.3 \%)$ & $0.033^{*}$ \\
Tea consumption & $36(33.0 \%)$ & $56(50.5 \%)$ & 0.153 \\
History of peptic ulcer & $4(3.7 \%)$ & $10(9.0 \%)$ & $3(2.7 \%)$ \\
Dual antiplatelet therapy & $10(9.2 \%)$ & $18(16.2 \%)$ & $0.000^{*}$ \\
Coumadin use & $1(0.9 \%)$ & $2(1.8 \%)$ & 0.966 \\
Nonaspirin NSAID use & $7(6.4 \%)$ & $29(26.1 \%)$ & 0.622 \\
Steroid use & $2(1.8 \%)$ & 1.000 \\
H. pylori infection & $52(47.7 \%)$ & $0.005^{*}$ \\
\hline
\end{tabular}

*denotes $P<0.05$.

gene promoter were classified into the following three groups: $\mathrm{T} / \mathrm{T}, \mathrm{T} / \mathrm{C}$, and $\mathrm{C} / \mathrm{C}$.

2.2.2. Statistical Analysis. Statistical evaluations were performed using the SPSS/Windows computer software package (Chicago, IL, USA). Two-sample $t$-tests were used to compare the mean values of the variables considered continuous in the peptic ulcer patients and controls. The chi-square test with or without Yate's correction for continuity and Fisher's exact test when appropriate were applied to analyze the categorized variables. Differences were considered to be significant at $P<0.05$. A stepwise forward conditional method was used to identify the independent risk factors for the development of peptic ulcer in low-dose aspirin users. The studied variables included the following: age $(<60$ or $\geq 60$ years), sex, blood type (O type or non-O type), history of smoking ( $<1$ pack/week or $\geq 1$ pack/week), history of alcohol consumption ( $<80 \mathrm{~g} /$ day or $\geq 80 \mathrm{~g} /$ day $), H$. pylori status (presence or absence), and genotype of COX-1 gene promoter.

According to a previous study [11], the frequency of $1676 \mathrm{~T}$ allele in the COX-1 gene promoter in healthy subjects was $47 \%$. We estimated that a $20 \%$ difference in the allele frequency could be present in low-dose aspirin users with peptic ulcer. Based on this assumption, 93 subjects had to be studied in each group to yield a statistical power of 0.80 and an $\alpha$ value of 0.05 .

\section{Results}

3.1. Clinical Characteristics of Aspirin Users with or without Peptic Ulcer. Two hundred and twenty aspirin users (126 from the Kaohsiung Veterans General Hospital and 94 from the Kaohsiung Medical University Hospital) were recruited for the study. They included 111 subjects with peptic ulcer disease (gastric ulcer: $n=90$; duodenal ulcer: $n=10$; both gastric and duodenal ulcers: $n=11$ ) and 114 controls.
Table 1 shows the demographic characteristics of peptic ulcer subjects and controls. The subjects with peptic ulcer were significantly older than the controls $(67.0 \pm 12.1$ versus $72.0 \pm$ $10.3 ; P=0.001)$. In addition, the rates of peptic ulcer history and the use of NSAID of peptic ulcer subjects were significantly higher than the controls ( $50.5 \%$ versus $3.7 \%$ and $16.2 \%$ versus $6.4 \%$, resp.; $P=0.000$ and $P=0.022$, resp.). However, the peptic ulcer group had a lower rate of coffee drinking than the control group (9.9\% versus $22.2 \% ; P=$ 0.033). H. pylori infection was detected in $26.2 \%$ of peptic ulcer subjects and in $47.5 \%$ of the controls. The infection rate was significantly lower in the peptic ulcer group than in the control group $(P=0.005)$. The two groups were similar with respect to gender, tea consumption, alcohol consumption, dual antiplatelet therapy with thienopyridine, and use of steroids.

\subsection{Genetic Factors Related to Peptic Ulcer Development in} Aspirin Users. Table 2 shows the blood group and genotype polymorphisms at position -1676 of the COX-1 gene promoter in aspirin users with and without peptic ulcer. The distributions of blood groups between aspirin users with and without peptic ulcer were different $(P=0.047)$. Peptic ulcer group had a higher rate of blood type $\mathrm{O}$ than the control group (45.9\% versus $29.4 \%$, resp.). The T/T, C/T, and C/C genotypes at position -1676 of the $C O X-1$ gene promoter in aspirin users with peptic ulcer were $24.7 \%, 66.3 \%$, and $9.0 \%$, respectively. No significant differences in genotype frequencies of the $C O X-1$ gene were found between the peptic ulcer group and control group $(P=0.245)$.

3.2.1. Independent Risk Factors for the Development of Peptic Ulcer in Aspirin Users. A stepwise forward logistic regression analysis for clinical and genetic variables was performed to search for the independent risk factors of peptic ulcer development in low-dose aspirin users. Multivariate analysis indicated that the advanced age, history of peptic ulcer, use of 
TABLE 2: Blood group and COX-1 genotype in low-dose aspirin users with and without peptic ulcer.

\begin{tabular}{|c|c|c|c|}
\hline & Gastritis/normal & Peptic ulcer & $P$ value \\
\hline Blood type & $(n=109)$ & $(n=111)$ & 0.047 \\
\hline A & $36(33.0 \%)$ & $29(26.1 \%)$ & \\
\hline B & $38(34.9 \%)$ & $25(22.5 \%)$ & \\
\hline $\mathrm{O}$ & $32(29.4 \%)$ & $51(45.9 \%)$ & \\
\hline $\mathrm{AB}$ & $5(4.6 \%)$ & $6(5.4 \%)$ & \\
\hline COX-1 genotype* & $(n=91)$ & $(n=95)$ & 0.245 \\
\hline $\mathrm{T} / \mathrm{T}$ & $15(19.5 \%)$ & $22(24.7 \%)$ & \\
\hline $\mathrm{C} / \mathrm{T}$ & $59(76.6 \%)$ & $59(66.3 \%)$ & \\
\hline $\mathrm{C} / \mathrm{C}$ & $3(3.9 \%)$ & $8(9.0 \%)$ & \\
\hline
\end{tabular}

${ }^{*}$ One hundred and eighty six DNA samples were available.

TABLE 3: Multivariate analysis for clinical and genetic factors related to the development of peptic ulcer in aspirin users.

\begin{tabular}{|c|c|c|c|c|}
\hline Clinical factor & Coefficient & Standard error & Odds ratio $(95 \% \mathrm{CI})$ & $P$ value \\
\hline Advanced age & 1.248 & 0.464 & $3.1(1.4-8.6)$ & 0.007 \\
\hline Peptic ulcer history & 3.320 & 0.576 & $27.6(8.9-85.5)$ & $<0.001$ \\
\hline Nonaspirin NSAID use & 1.154 & 0.525 & $2.9(1.1-8.0)$ & 0.045 \\
\hline Blood type O & 0.751 & 0.351 & $2.1(1.1-4.2)$ & 0.032 \\
\hline
\end{tabular}

NSAID, and blood type $\mathrm{O}$ were the independent risk factors for the development of peptic ulcer (Table 3 ). The odds ratios of the four parameters were $3.1,27.6,2.9$, and 2.1 , respectively (95\% confidence intervals, 1.4-8.6, 8.9-85.5, 1.1-8.0, and 1.14.2, resp.).

\section{Discussion}

Aspirin, usually available as an over-the-counter drug, is one of the most used drugs worldwide. In Western countries, 6 to $12 \%$ of the general population is exposed to low-dose aspirin $[15,16]$. It is well known that aspirin use is associated with upper gastrointestinal adverse effects [17]. However, only a small proportion of low-dose aspirin users develop peptic ulcer or life-threatening ulcer bleeding. Currently, the main genetic risk factors determining the development of peptic ulcer in low-dose aspirin remain unclear and pose a fascinating challenge in gastroenterology. In the current study, we investigate the clinical and genetic factors related to the development of peptic ulcer in low-dose aspirin users. The data indicated that the blood type $\mathrm{O}$, advanced age, history of peptic ulcer, and use of NSAID were independent factors predictive of ulcer development in low-dose aspirin users. The C-1676T polymorphism in the COX-1 gene promoter is not a risk factor for ulcer formation during treatment with low-dose aspirin.

Blood group $\mathrm{O}$ has traditionally been associated with the risk of developing peptic ulcer $[18,19]$. However, it remains unclear whether a subject with blood group $\mathrm{O}$ is a genetic marker for ulcer development during low-dose aspirin use. In this study, peptic ulcer group had a higher rate of blood type $O$ than that of the control group (45.9\% versus $29.4 \%$ ). Multivariate analysis with logistic regression documented that blood type $\mathrm{O}$ was an independent factor for ulcer development in low-dose aspirin users with an odds ratio of
2.1 (95\% CI: 1.1-4.2). Our study is the first in identifying blood group $\mathrm{O}$ as a genetic risk factor of peptic ulcer formation during treatment with low-dose aspirin.

Cyclooxygenases catalyze the conversion of arachidonic acid to prostaglandins. As a corollary, COX gene polymorphisms could be important in the pathogenesis of peptic ulcer disease because the function of cyclooxygenases affects prostaglandin formation and its protective effect at the level of the gastric mucosa. A previous study showed that the A-842G polymorphism did not play a significant role in the development of ulcer in NSAID users in the Japanese population [11]. In this study, we investigated the association between genetic polymorphisms in the COX-1 gene promoter and peptic ulcer development in low-dose aspirin users. Our data indicated that no significant differences in genotype frequencies of the C-1676T polymorphism in the COX-1 gene promoter were found between the peptic ulcer group and control group. However, Arisawa et al. reported that the C$1676 \mathrm{~T}$ functional polymorphism in the COX-1 gene promoter was related to the development of NSAID-induced ulcer [11]. The reasons for the contradictory results are unclear, but different doses of NSAIDs (low-dose aspirin for cardiovascular protection versus regular-dose NSAIDs for various neuromuscular diseases) or different ethnic populations are possible explanations.

In this study, we confirmed that advanced age, history of peptic ulcer, and use of NSAID were independent factors predictive of ulcer development in low-dose aspirin users. History of peptic ulcer is the most important clinical risk factor for ulcer development in low-dose aspirin use with an odds ratio of 27.6. The finding was consistent with previous studies that also identified a history of peptic ulcer as a clinical risk factor for peptic ulcer in low-dose aspirin users $[20,21]$. PPI has been shown to effectively reduce the risk of developing peptic ulcers associated with the continuous use 
of low-dose aspirin $[22,23]$. Therefore, cotherapy with a PPI to prevent the development of ulcers and ulcer complications could be considered in the aged patients with a previous history of peptic ulcer $[24,25]$.

Several other independent studies [26, 27] have also highlighted the importance of age factor in ulcer formation. García-Rodríguez and Jick [26] proved that advanced age increases risk of ulcer complications in an NSAID user. Lanas and Scheiman [4] also demonstrated that advanced age was an independent risk factor of ulcer formation in low-dose aspirin users. The reason why the aged stomach is more vulnerable to injury remains unclear. However, aging-related changes in gastric mucosa defense are possible explanations. Two human studies $[28,29]$ have demonstrated that gastric mucosal prostaglandin content declines with age. Feldman and Cryer [28] have also disclosed that advanced age is associated with a significant decline in gastric bicarbonate, sodium ion, and nonparietal fluid secretion. Thus, aging is associated with selective and specific changes in the gastric mucosal defenses that may predispose aged aspirin users to develop peptic ulceration.

In the current study, H. pylori infection was detected in $26.2 \%$ of peptic ulcer subjects and in $47.5 \%$ of the controls. The infection rate was significantly lower in the peptic ulcer group than in the control group. The reason for the lower $H$. pylori infection rate in the peptic ulcer group was probably due to prior anti-H. pylori therapy in many aspirin users in this patient group. Among the patients in peptic ulcer group, $50.5 \%$ of the subjects had a history of prior peptic ulcer. Multivariate analysis indicated that the history of peptic ulcer was the independent risk factor for the development of peptic ulcer and $H$. pylori status was removed from independent factors related to peptic ulcer development following logistic regression analysis.

The strengths of this study included investigating both clinical and genetic risk factors for ulcer development in lowdose aspirin users and prospective assessment of clinical data of the recruited subjects. However, it has several limitations. First, the sample size was moderate and possibly unable to detect minor independent risk factors for the development of peptic ulcer in low-dose aspirin users. Secondly, aspirin users who took PPIs or histamine-2 receptor antagonists before endoscopy were excluded. We therefore could not identify some protective factors for ulcer development in low-dose aspirin users. Thirdly, it was a case-control study and selection bias of recruited cases could not be completely ruled out. A large-scale, long-term cohort study is therefore merited to clarify the risk factors identified in this work.

In conclusion, blood type $\mathrm{O}$, advanced age, history of peptic ulcer, and use of nonaspirin NSAID are independent risk factors for development of peptic ulcer in low-dose aspirin users. The $\mathrm{C}-1676 \mathrm{~T}$ polymorphism in the $C O X-1$ gene promoter is not a risk factor for ulcer formation during treatment with low-dose aspirin.

\section{Conflict of Interests}

The authors declare that there is no conflict of interests regarding the publication of this paper.

\section{Acknowledgments}

The study was partly supported by a research grant from the Kaohsiung Veterans General Hospital, Kaohsiung, Taiwan (VGHKS100-094). The authors are indebted to Drs. H. M. Wang and J. L. Ou for recruiting the patients and performing the endoscopies and study nurse L. Y. Wang at the Kaohsiung Veterans General Hospital.

\section{References}

[1] Steering Committee of the Physicians' Health Study Research Group, "Final report on the aspirin component of the ongoing physician's health study," The New England Journal of Medicine, vol. 321, no. 3, pp. 129-135, 1989.

[2] T. A. Pearson, S. N. Blair, S. R. Daniels et al., "AHA guidelines for primary prevention of cardiovascular disease and stroke: 2002 update: consensus panel guide to comprehensive risk reduction for adult patients without coronary or other atherosclerotic vascular diseases," Circulation, vol. 106, no. 3, pp. 388-391, 2002.

[3] U. A. Ajani, E. S. Ford, K. J. Greenland, W. H. Giles, and A. H. Mokdad, "Aspirin use among U.S. adults: behavioral risk factor surveillance system," The American Journal of Preventive Medicine, vol. 30, no. 1, pp. 74-77, 2006.

[4] A. Lanas and J. Scheiman, "Low-dose aspirin and upper gastrointestinal damage: epidemiology, prevention and treatment," Current Medical Research and Opinion, vol. 23, no. 1, pp. 163173, 2007.

[5] B. Cryer and M. Feldman, "Effects of very low dose daily, long-term aspirin therapy on gastric, duodenal, and rectal prostaglandin levels and on mucosal injury in healthy humans," Gastroenterology, vol. 117, no. 1, pp. 17-25, 1999.

[6] L. Laine, "Approaches to nonsteroidal anti-inflammatory drug use in the high-risk patient," Gastroenterology, vol. 120, no. 3, pp. 594-606, 2001.

[7] G. V. Moukarbel, J. E. Signorovitch, M. A. Pfeffer et al., "Gastrointestinal bleeding in high risk survivors of myocardial infarction: the VALIANT Trial," European Heart Journal, vol. 30, no. 18 , pp. 2226-2232, 2009.

[8] G. Edgren, H. Hjalgrim, K. Rostgaard et al., "Risk of gastric cancer and peptic ulcers in relation to $\mathrm{ABO}$ blood type: a cohort study," American Journal of Epidemiology, vol. 172, no. 11, pp. 1280-1285, 2010.

[9] M. K. Halushka, L. P. Walker, and P. V. Halushka, "Genetic variation in cyclooxygenase 1: effects on response to aspirin," Clinical Pharmacology and Therapeutics, vol. 73, no. 1, pp. 122130, 2003.

[10] T. Heinemeyer, E. Wingender, I. Reuter et al., "Databases on transcriptional regulation: TRANSFAC, TRRD and COMPEL," Nucleic Acids Research, vol. 26, no. 1, pp. 362-367, 1998.

[11] T. Arisawa, T. Tahara, T. Shibata et al., "Association between genetic polymorphisms in the cyclooxygenase-1 gene promoter and peptic ulcers in Japan," International Journal of Molecular Medicine, vol. 20, no. 3, pp. 373-378, 2007.

[12] P. I. Hsu, K. H. Lai, and C. P. Liu, "Esomeprazole with clopidogrel reduces peptic ulcer recurrence, compared with clopidogrel alone, in patients with atherosclerosis," Gastroenterology, vol. 140, no. 3, pp. 791-798, 2011.

[13] C. Liu, W. Chen, K. Lai et al., "Esomeprazole alone compared with esomeprazole plus aspirin for the treatment of aspirinrelated peptic ulcers," The American Journal of Gastroenterology, vol. 107, no. 7, pp. 1022-1029, 2012. 
[14] A. Shiotani, T. Sakakibara, Y. Yamanaka et al., "The preventive factors for aspirin-induced peptic ulcer: aspirin ulcer and corpus atrophy," Journal of Gastroenterology, vol. 44, no. 7, pp. 717-725, 2009.

[15] P. Czernichow and V. Merle, "Epidemiology of digestive complications associated with use of low-dose aspirin," Gastroentérologie Clinique et Biologique, vol. 28, no. 3, pp. C37-C44, 2004.

[16] M. G. Miller, B. D. Lucas Jr., V. Papademetriou, and A. Elhabyan, "Aspirin under fire: aspirin use in the primary prevention of coronary heart disease," Pharmacotherapy, vol. 25, no. 6, pp. 847-861, 2005.

[17] P. I. Hsu, "New look at antiplatelet agent-related peptic ulcer: an update of prevention and treatment," Journal of Gastroenterology and Hepatology, vol. 27, no. 4, pp. 654-661, 2012.

[18] C. A. Clarke, J. W. Edwards, D. R. Haddock, A. W. Howel-Evans, R. B. McConnell, and P. M. Sheppard, "ABO blood groups and secretor character in duodenal ulcer. Population and sibship studies," British Medical Journal, vol. 2, no. 4995, pp. 725-731, 1956.

[19] H. O. Hein, P. Suadicani, and F. Gyntelberg, "Genetic markers for peptic ulcer. A study of 3387 men aged 54 to 74 years: the Copenhagen Male Study," Scandinavian Journal of Gastroenterology, vol. 32, no. 1, pp. 16-21, 1997.

[20] P. Serrano, A. Lanas, M. T. Arroyo, and I. J. Ferreira, "Risk of upper gastrointestinal bleeding in patients taking low-dose aspirin for the prevention of cardiovascular diseases," Alimentary Pharmacology \& Therapeutics, vol. 16, no. 11, pp. 1945-1953, 2002.

[21] J. Iwamoto, Y. Saito, A. Honda, and Y. Matsuzaki, "Clinical features of gastroduodenal injury associated with long-term low-dose aspirin therapy," World Journal of Gastroenterology, vol. 19, no. 11, pp. 1673-1682, 2013.

[22] K. C. Lai, S. K. Lam, K. M. Chu et al., "Lansoprazole for the prevention of recurrences of ulcer complications from long-term low-dose aspirin use," The New England Journal of Medicine, vol. 346, no. 26, pp. 2033-2038, 2002.

[23] N. Yeomans, A. Lanas, J. Labenz et al., "Efficacy of esomeprazole (20 mg once daily) for reducing the risk of gastroduodenal ulcers associated with continuous use of low-dose aspirin," The American Journal of Gastroenterology, vol. 103, no. 10, pp. 24652473, 2008.

[24] F. K. L. Chan, "Primer: Managing NSAID-induced ulcer complications-balancing gastrointestinal and cardiovascular risks," Nature Clinical Practice Gastroenterology and Hepatology, vol. 3, no. 10, pp. 563-573, 2006.

[25] D. L. Bhatt, J. Scheiman, N. S. Abraham et al., "ACCF/ACG/ AHA 2008 Expert Consensus Document on Reducing the Gastrointestinal Risks of Antiplatelet Therapy and NSAID Use. A Report of the American College of Cardiology Foundation Task Force on Clinical Expert Consensus Documents," Journal of the American College of Cardiology, vol. 52, no. 18, pp. 15021517, 2008.

[26] L. A. García Rodríguez and H. Jick, "Risk of upper gastrointestinal bleeding and perforation associated with individual nonsteroidal anti-inflammatory drugs," The Lancet, vol. 343, no. 8900, pp. 769-772, 1994.

[27] R. K. Simons, D. B. Hoyt, R. J. Winchell et al., "A risk analysis of stress ulceration after trauma," Journal of Trauma: Injury Infection \& Critical Care, vol. 39, no. 2, pp. 289-294, 1995.
[28] M. Feldman and B. Cryer, "Effects of age on gastric alkaline and nonparietal fluid secretion in humans," Gerontology, vol. 44, no. 4, pp. 222-227, 1998.

[29] P. Maity, K. Biswas, S. Roy, R. K. Banerjee, and U. Bandyopadhyay, "Smoking and the pathogenesis of gastroduodenal ulcer: recent mechanistic update," Molecular and Cellular Biochemistry, vol. 253, no. 1-2, pp. 329-338, 2003. 


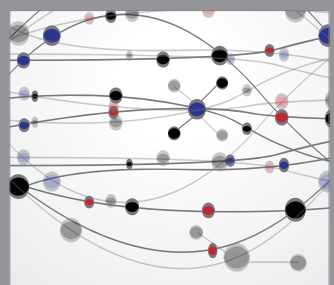

The Scientific World Journal
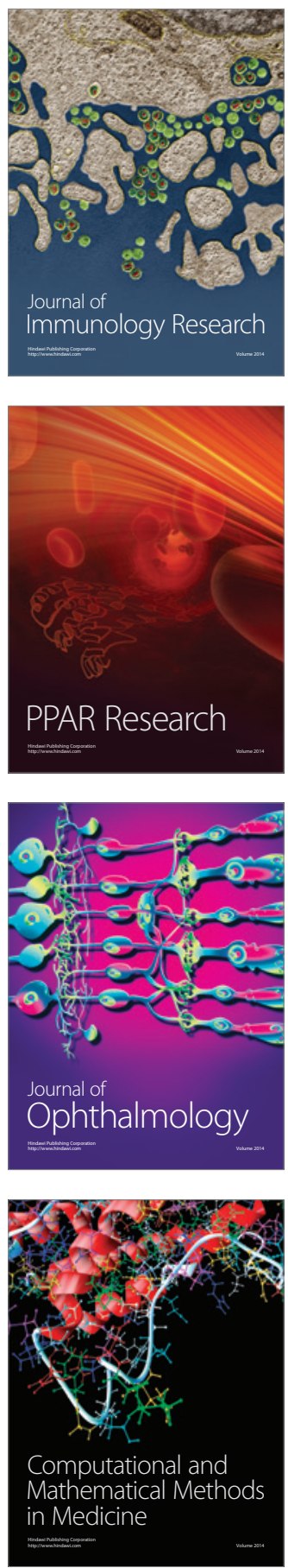

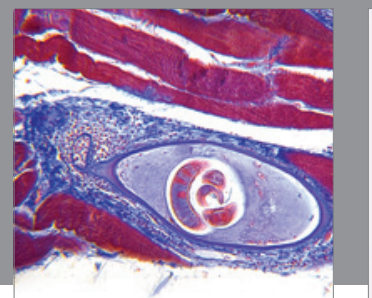

Gastroenterology

Research and Practice
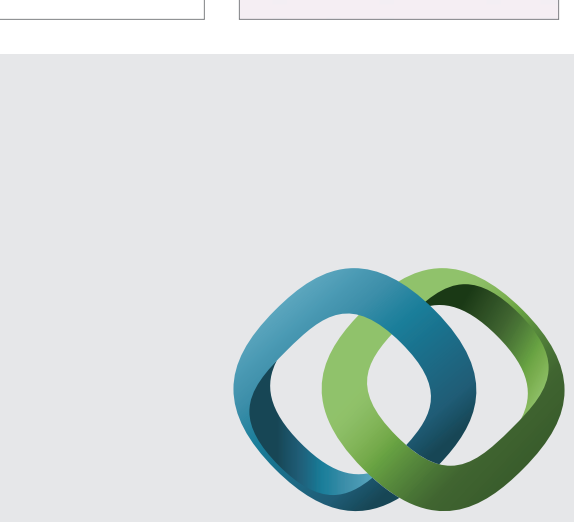

\section{Hindawi}

Submit your manuscripts at

http://www.hindawi.com
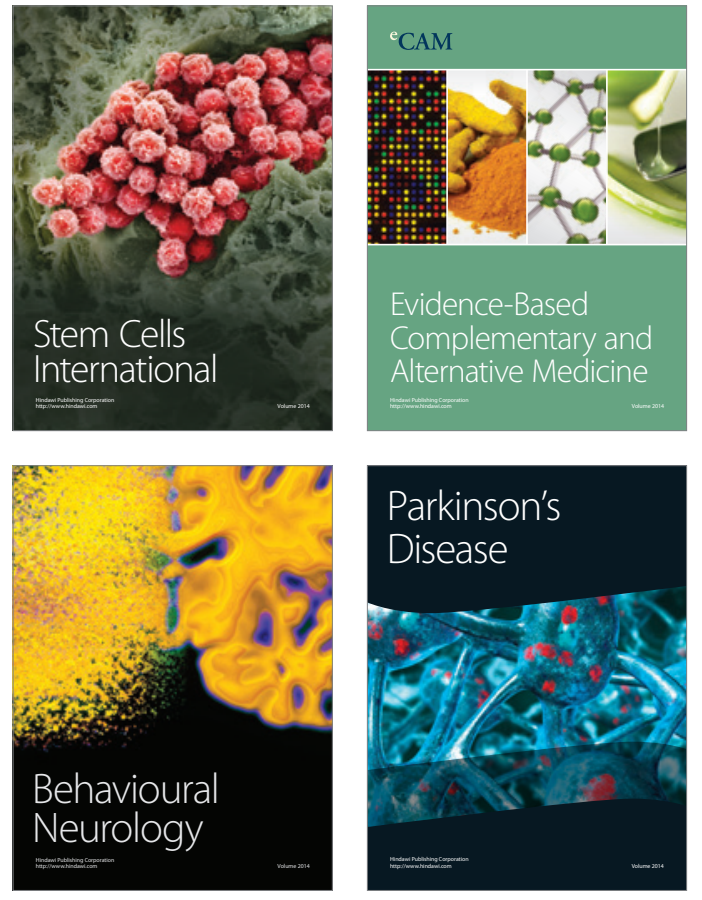
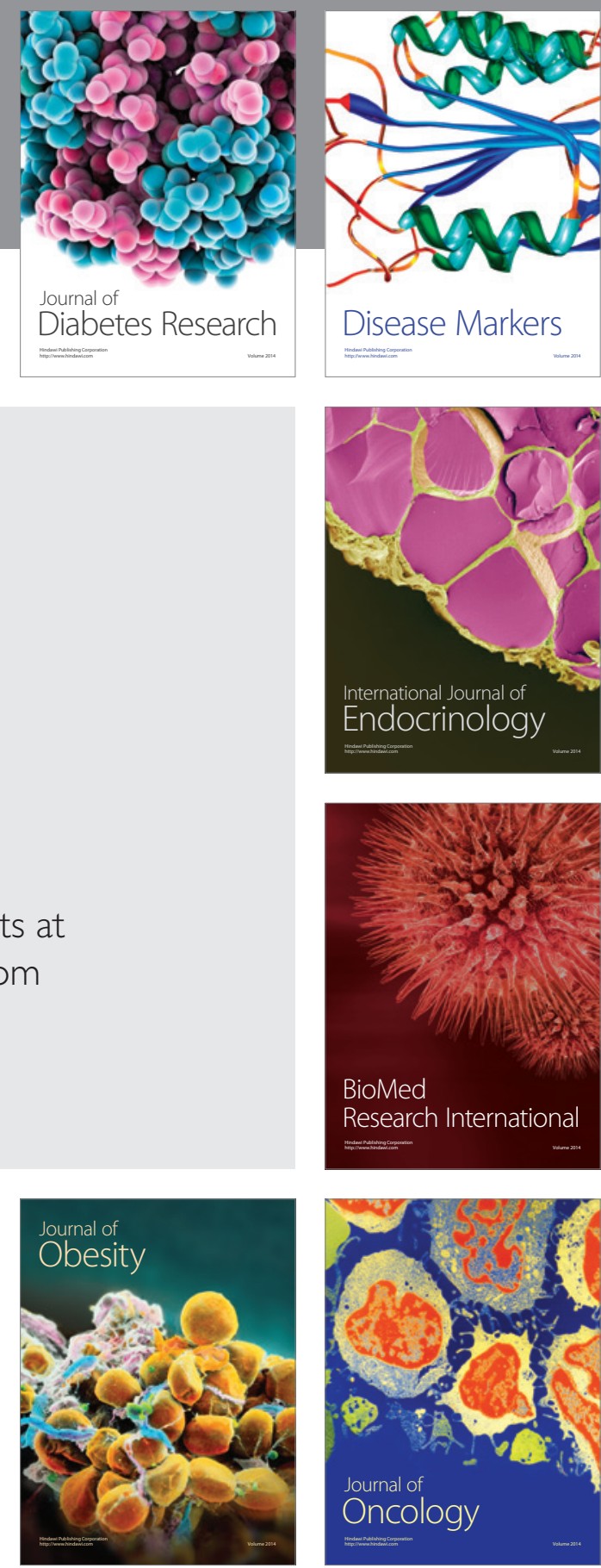

Disease Markers
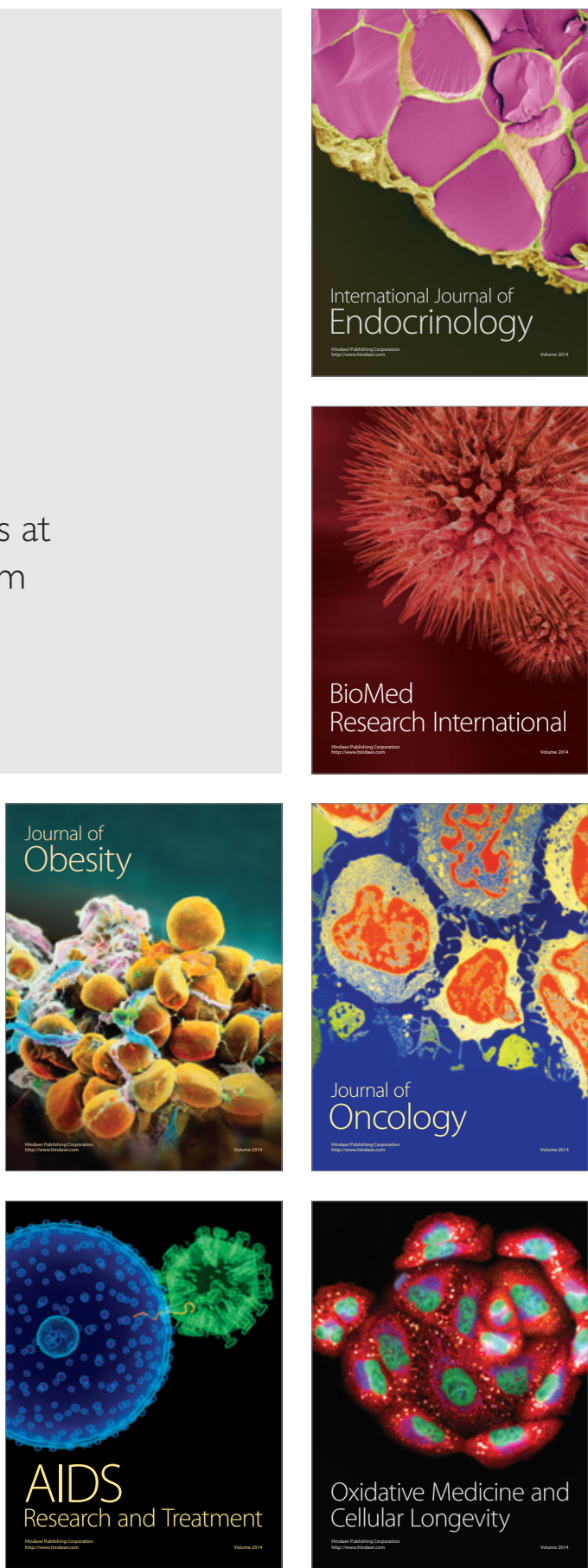